\title{
Magnetic Field Measurement and Compensation in the Recycler Electron Cooler
}

\author{
V.Tupikov $^{\# 1}$, G..Kazakevich ${ }^{2}$, T.K.Kroc ${ }^{1}$, S.Nagaitsev ${ }^{1}$, L.Prost ${ }^{1}$, \\ A.Shemyakin ${ }^{1}$, C.W.Schmidt ${ }^{1}$, M.Sutherland ${ }^{1}$, A. Warner $^{1}$ \\ ${ }^{1}$ FNAL, Batavia, IL 60510, USA \\ ${ }^{2}$ BINP, Novosibirsk, 630090, Russia
}

\begin{abstract}
Cooling of 8.9-GeV/c antiprotons in the Recycler Electron Cooler requires a round 4.34-MeV electron beam with a small angular spread propagating through a $20-\mathrm{m}$ long cooling section. To confine the electron beam tightly and to keep its total transverse angles below 0.2 mrad the cooling section is immersed in a solenoidal field of 50-200 G. The field was measured with a compass-based sensor (transversal) and a hall-probe (longitudinal) after installation of the solenoids into the Recycler tunnel. For the field strength of $105 \mathrm{G}$, the transverse field components were compensated to the level that provided corresponding dipole beam oscillations below $0.1 \mathrm{mrad}$, which in turn allowed the first cooling of antiprotons in the $\mathrm{GeV}$ energy range. This paper discusses the field measurements and compensation scheme including the results of dipole oscillation measurements.
\end{abstract}

Keywords: Electron Cooling.

COOL: 05

\section{INTRODUCTION}

The Electron Cooling project at Fermilab is characterized by a low 100-G magnetic field in the cooling section (CS), despite conventional coolers with kilogauss range fields. As a result, the helical length, $\lambda$, of an electron in the CS for a closed Larmor radius becomes much longer ( $\lambda \gg \mathrm{d}_{\text {SOLENOID }}$ ), which makes a low value of $\int B_{\perp} d z$ more important then an absolute low value of $B_{\perp}$. The system used for magnetic measurements has been discussed in previous publications [1-2]. The cooling section solenoid design is discussed in [3].
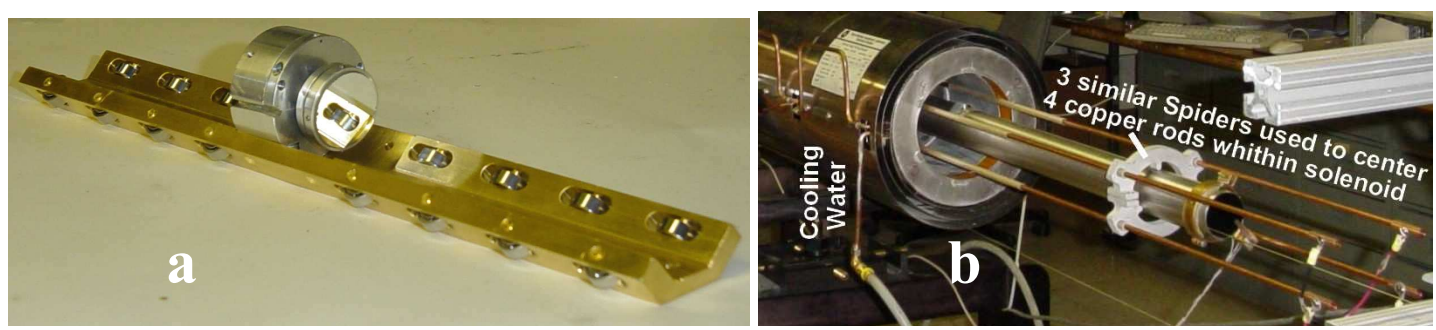

Figure 1 (a) New wheeled cart design with compass. Compass is replaced by hall-probe during longitudinal measurements; (b) Four copper rods compensator. 


\section{MAGNETIC MEASUREMENTS}

\section{Preliminary Test Bench Run}

Test bench measurements for each of 10 of the cooling section solenoids preceded the final measurements in the Recycler tunnel. They resulted in a field map for each 2$\mathrm{m}$ long solenoid modules. The map consists of longitudinal and transverse field components. The longitudinal component is a product of the "main" and two "trim" solenoids, measured separately. The transverse component represents the field generated by compensation coils, which are ten $\mathrm{X}$ and $\mathrm{Y}$ pairs per module. The field shape generated by the compensation coil is well described by a Gaussian distribution with the center given with respect to a "zeroed" longitudinal lead trim-solenoid peak.

Following the idea of the BINP (Novosibirsk) design, the transverse field measurement scheme had been revised in comparison to the one described in [1-2]. We omitted the cylindrical cart design with wrapped compensation coils because of its deficiencies: unpredictable $\mathrm{X}$ and $\mathrm{Y}$ component coupling caused by cart rotation; occasional cart sticking in the chamber; and jerky movement. The new design, based on a wheeled cart (Figure 1a) and 4-rod compensator (Figure 1b) was a significant improvement and-removed the sticks, jerks, and rotations. The cart movement became smoother giving precise transverse measurements due to elimination of the cable connection.

\section{Longitudinal Field}

All longitudinal field measurements were done with use of a BH204 hall-probe from "F.W.Bell Co". The initial probe calibration was done against a calibrated Teslameter DTM-141 at the Fermilab Technical Department's magnetic measurement test bench. The achieved $0.1 \%$ calibration accuracy was crucial not only for magnetic field mapping but possibility for matching the energies of electron and antiproton beams as well. For procedure details refer to [4] and to [5] for how the matching was successfully achieved.
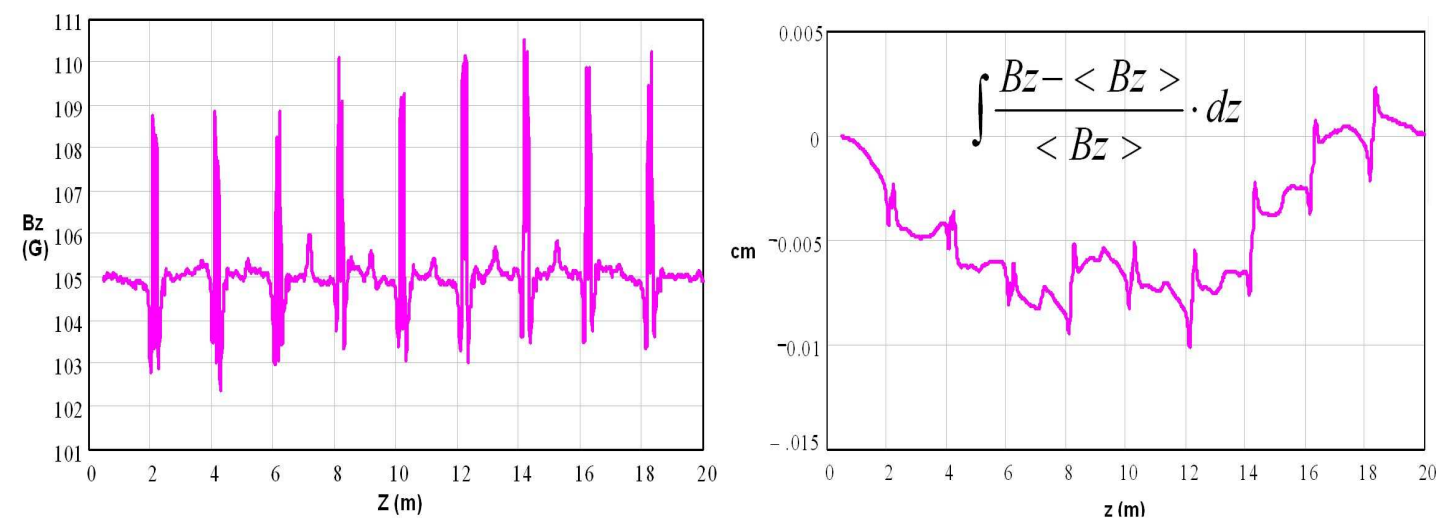

Figure $2 \mathrm{Bz}$ measurement after solenoid current correction (left plot). Integral of Bz field deviation from the average with $\angle \mathrm{Bz}>=105.1 \mathrm{G}$ (right plot). 

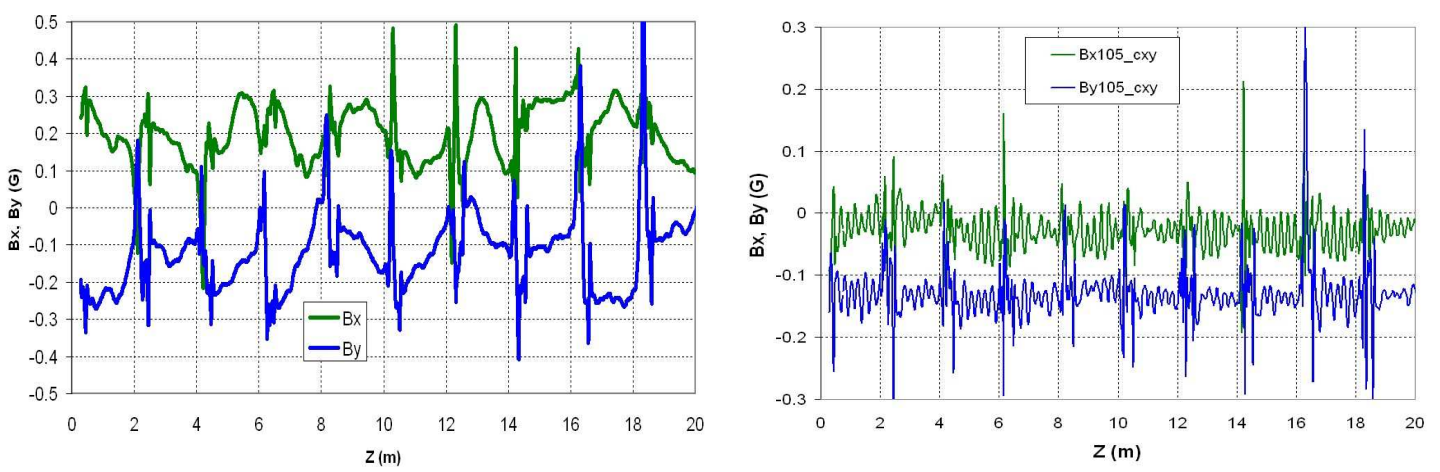

Figure 3 Left plot - First measurement of transverse field taken at $\langle\mathrm{Bz}>=105.1$ G. Right plot Second measurements after field correction by dipoles. There is a large By-offset, caused by a broken wire between initial and second measurements.

The first measurements were made with the lead trim-solenoids on and the return trim- and main solenoids off. It permitted defining dipole elements to the absolute Zcoordinate in the Recycler tunnel, based on dipole peaks, with coordinates for each solenoid found during preliminary measurements.

Then, longitudinal field was measured along the full cooling section (CS) at the expected nominal settings for solenoid currents. Due to varying winding numbers for each solenoid during manufacturing, the fields generated by the solenoid differed slightly. The field was divided into the 'main solenoid' and 'gap' intervals, where the average field values were calculated. Each 'main solenoid' is powered by an individual power supply, but all trim-solenoids share just two power supplies feeding lead- and return- solenoids connected in series respectively. The equalization of the 'main solenoid' averages were done by adjusting DAC settings for their individual power supplies. In the case of averages for trim-solenoids, - the equalizations were achieved by resistive shunts connected in parallel to the coils to bypass some current.

The final longitudinal field corresponding to $105 \mathrm{G}$ average is shown in Figure 2. The plot of Bz integral deviation along the CS is less than $0.1 \mathrm{~cm}$ which is one order of magnitude better than cooling requirements.

For possibly working at different $\mathrm{Bz}$ values, a set of measurements of $\mathrm{Bz}(\mathrm{z})$ versus trim-solenoid currents in more problematic regions (gaps), were made. Then, calculated integrals of bell-like fields as a function of induced current were plotted, and were perfectly linear. Having measured the longitudinal field in the CS at $190 \mathrm{G}$, we are now able to approximate field settings for any field from $50 \mathrm{G}$ through $190 \mathrm{G}$.

\section{Transverse Field}

The first transverse measurements started at longitudinal field $\mathrm{Bz}=105 \mathrm{G}$ and all dipole correctors set to zero current (Figure 3, left plot). The choice of 105-G field was defined by the period of the electron's Larmore radius. In a case of $100 \mathrm{G}$ longitudinal field it is very close to an integer number of BPM placements in the CS, which makes difficult to make a field correction by zeroing the electron beam in BPMs as it will be described in next chapter.

The measured field map combined from longitudinal and transverse components was entered into a simulation program, which calculated the dipole corrector values 
necessary to compensate the transverse field in the CS. Corrected currents were applied to the correctors and a second run of transverse measurement were taken. Again, the discrepancy in the transverse field between measured (during the second run) and expected field (superposition of fields measured during the first run and calculation of field generated by dipole corrector settings found after first run) was simulated. Corrector changes were found for this discrepancy and applied to the settings of the first run. The results of the composite measurements is shown in Figure 3 on the right plot, where dark color curves present measured transverse components. The calculated electron angles in the CS were equal to $50 \mu \mathrm{rad}$, disregarding offsets; mostly determined by residual tension of the compass suspension and unresolved during the measurements.

Transverse fields at $\mathrm{Bz}=190 \mathrm{G}$ was measured once- and gave a reduction of field compensation accuracy. The estimated electron angles in the CS at this field were 130 $\mu \mathrm{rad}$.

\section{Further Field Correction By Electron Beam Position (BPM)}

After the Pelletron was commissioned and first beam in the CS was obtained, additional field improvement was achieved. In this case, corrections made to the dipole settings relied on beam position monitor (BPM) readings. All CS BPMs had been very precisely calibrated against the pbar beam.

First attempt was to get rid of unresolved transverse field offsets. Monitoring the ebeam trajectory in the CS and minimizing the trajectory oscillation required tuning the current, by the same value, of all of horizontal and then vertical dipole correctors. The current changes of $170 \mathrm{~mA}$ for horizontal and $-215 \mathrm{~mA}$ for vertical planes led to an electron trajectory with $1 \mathrm{~mm}$ peak-to-peak oscillation in the CS. Then, changing the current by the same value in all of the correctors (separately for X and Y planes) of any $2 \mathrm{~m}$-long section gave a zeroed beam displacement in the closest downstream BPM (Figure 4). Due to a ground fault in CXC80 and CYC80 correctors $20 \%$ of the CS was lost from effective cooling (see electron trajectory after $16 \mathrm{~m}$ on the plot), but we are going to get it back by replacing the regular power supplies with isolated outputs.
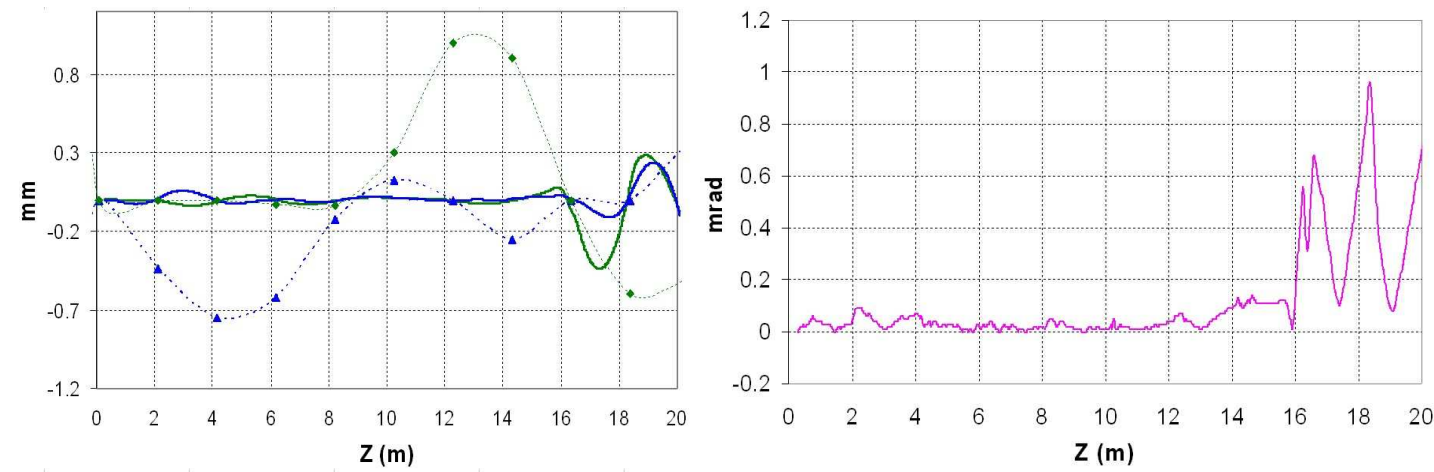

Figure 4. Left plot - Electron trajectories in CS before (dash curves) and after (solid curves) section by section adjustment Right plot - Estimated electron angles in CS after compensation. 
Transverse field correction was done at a longitudinal field of $190 \mathrm{G}$ as well. Now it is possible to compensate for any longitudinal field in the range of 105 to $190 \mathrm{G}$. New corrector settings were calculated by formula:

$$
C_{X\{Y\}}^{150 G}=C_{X\{Y\}}^{105 G}+\frac{(150-105) G}{(190-105) G}\left(C_{X\{Y\}}^{190 G}-C_{X\{Y\}}^{105 G}\right)
$$

where $\mathrm{C}$ is the corrector current .

With these settings, and no additional tuning, the beam angle in the CS was estimated to be $130 \mu \mathrm{rad}$. This is the same number found for the compensated field at $\mathrm{Bz}=190 \mathrm{G}$, thus it is sort of an error translation.

\section{SUMMARY}

The magnetic field in the cooling section was measured for both transverse and longitudinal components. Then the transverse filed was compensated with dipole correctors. Good cooling requires electron angles in the CS to be less than $100 \mu \mathrm{rad}$. The first estimation of electron angles in the CS with use of a simulation program and the compensated field map gave $50 \mu \mathrm{rad}$. The second estimation, by drag force measurements, gave $100 \mu \mathrm{rad}[6]$.

\section{ACKNOWLEDGEMENTS}

The authors would like to express appreciations to V.Parkhomchuk, whose ideas made the measurement system possible; J.Leibfritz, V.Sidorov and L.Nobrega for their engineering support; K.Carlson - for electrical engineering help; and the teams of technicians and electricians working with us, especially: R.Kellet, A.Germain, J.Nelson, W.Johnson.

\section{REFERENCES}

1. The Precise Magnetic Field Sensor for Solenoids of Cooling Section, V.N.Bocharov et al, Proc. Of XVII Workshop on Particle Accelerators, Protvino, Russia, October 17-20, 2000 (in Russian).

2. Field Measurements In The Fermilab Electron Cooling Solenoid Prototype, V.Tupikov, et.al., FermilabTM-2224, October, 2003.

3. Fermilab Electron Cooling Project: Estimates for the Cooling Section Solenoid, Fermilab-FN-689, S.Nagaitsev, A.Shemyakin and V.Vostrikov, April 2000.

4. Beam-Based Calibration of the Electron Energy in the Fermilab Electron Cooler, S. M. Seletskiy, A. Shemyakin; Proceedings of PAC2005, Knoxville, TN, USA, May 16-20, 2005.

5. Attainment of high-quality electron beam for Fermilab 4.3-MV cooler, A.Shemyakin, et al., This Workshop Proceedings.

6. Antiproton cooling in the Fermilab Recycler, S.Nagaitsev, This Workshop Proceedings. 